\title{
Simulation of an Autonomous Biped Walking Robot Including Environmental Force Interaction
}

\author{
Yasutaka Fujimoto*, Member, IEEE, Atsuo Kawamura**, Senior Member, IEEE
}

\begin{abstract}
This autonomous biped walking control system is based on the reactive force interaction at the foothold. The precise 3D (three dimensional) dynamic simulation presented includes: 1) a posture controller which accommodate the physical constraints of the reactive force/torque on the foot by quadratic programming. 2) a real-time COM (center of mass) tracking controller for foot placement, with a discrete inverted pendulum model. 3) a 3D dynamic simulation scheme with precise contact with the environment. The proposed approach realizes the robust biped locomotion because the environmental interaction is directly controlled. The proposed method is applied to the 20 axes simulation model, and the stable biped locomotion with velocity of 0.25 $\mathrm{m} / \mathrm{sec}$ and stepping time of $0.5 \mathrm{sec} / \mathrm{step}$ is realized.
\end{abstract}

\section{INTRODUCTION}

A NUMBER OF biped walking systems have been proposed in the previous works[1]-[12]. Since the reactive force and torque on the foothold depend on its complicated characteristics, the relation between the input torque of the actuator and the reactive force and torque is hard to solve. Thus the conventional control systems calculate trajectories of joint angle or joint torque so as to approximately satisfy the stable contact condition[1]-[3]. The approximation, however, yields lack of walking robustness. The whole dynamic equation of the robot and the contact condition is considered to generate joints references in [3], but it is off-line type planning due to the complexity of dynamics of the biped robot.

In this paper, a new hierarchical control system based on the reactive force control on the foothold and the force distribution system is proposed in order to improve the walking robustness, in which the physical constraints of the contact force on the foothold are precisely considered. With a new algorithm of the foot placement, an on-line controlled autonomous biped locomotion is realized. Also, the precise 3D dynamic simulator with the environmental interaction is proposed to investigate the control scheme.

\section{Modeling}

A legged robot is modeled as a free-fall manipulator which has no fixed-point but has interaction to the ground. A dynamics of a free-falling manipulator is formulated by introducing the variables representing position and attitude of a base-link. Let generalized coordinates $\boldsymbol{x}$, generalized

*Department of System Design Engineering, Keio University, Yokohama 223-8522, Japan. **Department of Electrical and Computer Engineering, Yokohama National University, Yokohama 240-8501 Japan. Part of this research is carried with a subsidy of the Scientific Research Fund of the Ministry of Education.

1070-9932/98/\$10.00@1998 IEEE velocities $\boldsymbol{v}$, and generalized forces $\boldsymbol{u}$ be

$$
\begin{aligned}
& \boldsymbol{x}^{T}=\left[\boldsymbol{p}_{B}^{T}, \boldsymbol{A}_{B}^{T}, \boldsymbol{\theta}^{T}\right] \in R^{3} \times S O(3) \times R^{N} \\
& \boldsymbol{v}^{T}=\left[\boldsymbol{v}_{B}^{T}, \boldsymbol{\omega}_{B}^{T}, \boldsymbol{\omega}^{T}\right] \in R^{3} \times R^{3} \times R^{N} \\
& \boldsymbol{u}^{T}=\left[\boldsymbol{f}_{B}^{T}, \boldsymbol{n}_{B}^{T}, \boldsymbol{\tau}^{T}\right] \in R^{3} \times R^{3} \times R^{N}
\end{aligned}
$$

where

$\boldsymbol{p}_{B}: 3 \times 1$ vector specifying base-link position

$\boldsymbol{A}_{B}: 3 \times 3$ matrix specifying base-link attitude

$\boldsymbol{\theta}: N \times 1$ vector specifying joint angle

$\boldsymbol{v}_{B}: 3 \times 1$ vector specifying base-link velocity

$\boldsymbol{\omega}_{B}: 3 \times 1$ vector specifying angular velocity of base-link

$\boldsymbol{\omega}: N \times 1$ vector specifying joint angular velocity

$f_{B}: 3 \times 1$ force vector generated in base-link

$n_{B}: 3 \times 1$ torque vector generated in base-link

$\tau: N \times 1$ torque vector generated by actuator

$N$ : number of joints of robot

$\boldsymbol{A}_{B}$ is the direction matrix of the inertial base-link-fixed axes relative to the ground-fixed axes. (See Fig. 1, $\boldsymbol{A}_{B}=$ $\left[\boldsymbol{x}_{B}, \boldsymbol{y}_{B}, \boldsymbol{z}_{B}\right]$.) The attitude $\boldsymbol{A}_{B}$ moves in the Lie group $S O(3)$.

The equations of motion of the robot become:

$$
\begin{aligned}
\dot{\boldsymbol{p}}_{B} & =\boldsymbol{v}_{B} \\
\dot{\boldsymbol{A}}_{B} & =\boldsymbol{\omega}_{B} \times \boldsymbol{A}_{B} \\
\dot{\boldsymbol{\theta}} & =\boldsymbol{\omega}
\end{aligned}
$$

and

$$
\boldsymbol{H}(\boldsymbol{x}) \dot{\boldsymbol{v}}+\boldsymbol{C}(\boldsymbol{x}, \boldsymbol{v}) \boldsymbol{v}+\boldsymbol{g}(\boldsymbol{x})=\boldsymbol{u}+\boldsymbol{u}_{E}
$$

where

$\boldsymbol{H}(\boldsymbol{x}):(N+6) \times(N+6)$ inertia matrix

$\boldsymbol{C}(\boldsymbol{x}, \boldsymbol{v}):(N+6) \times(N+6)$ matrix specifying centrifugal and Corioli's effects

$\boldsymbol{g}(\boldsymbol{x}):(N+6) \times 1$ vector specifying gravity effect

$\boldsymbol{u}_{E} \quad:(N+6) \times 1$ vector specifying generalized forces generated by external forces

\section{Control Algorithm}

To control a legged robot (4)-(7), we must consider physical constraints on the foothold directly or indirectly. In this paper, a hierarchical control system with a direct and real-time method is proposed, which realizes the robust contact of the foothold and the stable biped locomotion. Fig. 2 shows the proposed hierarchical system. The lower layer of the system consists of a workspace position controller and a robust reactive force controller. 


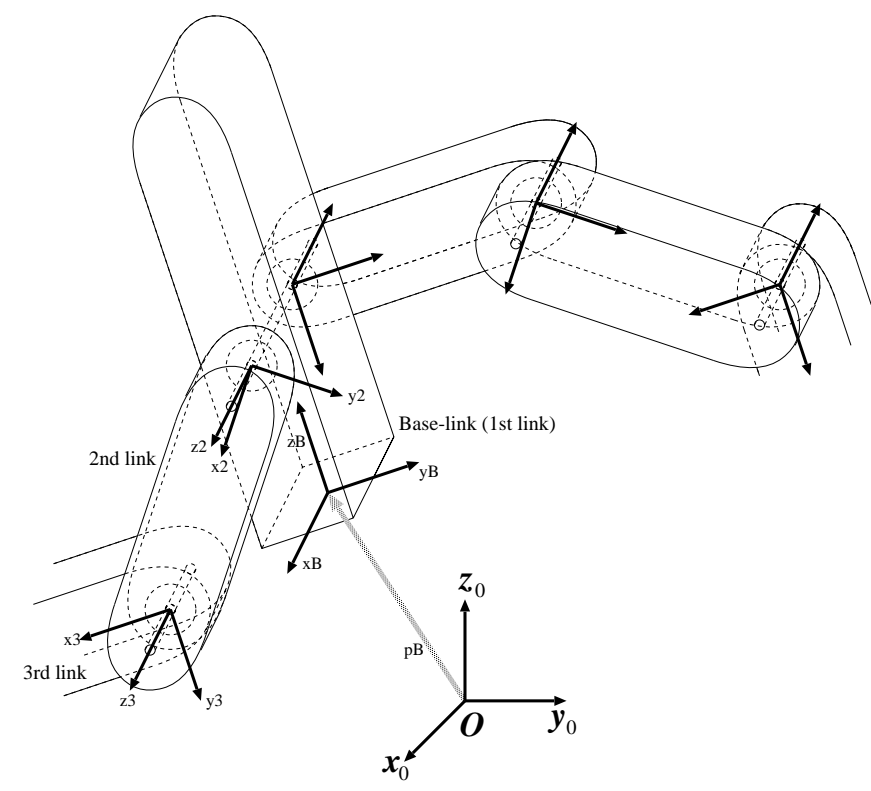

Fig. 1. Representation of link-fixed coordinates.

\section{A. Posture Controller}

In a case of a biped robot, $\boldsymbol{u}_{E}$, the external force on the generalized coordinates, is expressed as follows.

$\boldsymbol{u}_{E}=\left[\begin{array}{cc}\boldsymbol{I}_{3} & \mathbf{0} \\ {\left[\boldsymbol{x}_{R} \times\right]} & \boldsymbol{I}_{3} \\ \boldsymbol{J}_{R 1}^{T} & \boldsymbol{J}_{R 2}^{T}\end{array}\right]\left[\begin{array}{c}\boldsymbol{f}_{R} \\ \boldsymbol{n}_{R}\end{array}\right]+\left[\begin{array}{cc}\boldsymbol{I}_{3} & \mathbf{0} \\ {\left[\boldsymbol{x}_{L} \times\right]} & \boldsymbol{I}_{3} \\ \boldsymbol{J}_{L 1}^{T} & \boldsymbol{J}_{L 2}^{T}\end{array}\right]\left[\begin{array}{c}\boldsymbol{f}_{L} \\ \boldsymbol{n}_{L}\end{array}\right]$

where

$\boldsymbol{f}_{R}, \boldsymbol{f}_{L}: 3 \times 1$ vector of reactive force at the center of right or left foot

$n_{R}, n_{L}: 3 \times 1$ vector of reactive torque at the center of right or left foot

$\boldsymbol{J}_{R i}, \boldsymbol{J}_{L i}: 3 \times N$ Jacobian matrix of right or left foot

$\boldsymbol{x}_{R}, \boldsymbol{x}_{L}: 3 \times 1$ position vector of the right or left foot with respect to the origin of $\boldsymbol{p}_{B}$

Here, $[\boldsymbol{a} \times]$ denotes a matrix representing a cross product, and $\boldsymbol{I}_{n}$ denotes an $n \times n$ identity matrix.

While there is no actuated control input for the position and the attitude of the body, i. e., $\boldsymbol{f}_{B}=\boldsymbol{n}_{B}=0$, those of the body are still controllable by using the reactive force and torque $\left(f_{R}, f_{L}, n_{R}, n_{L}\right)$ as indirect control inputs. To use the reactive force and torque as indirect control inputs, the hybrid position/force control is applied to each leg. If the leg is in the support phase, the force control is activated. Otherwise the position control becomes active. The workspace position control system consists of the inertia fluctuation insensitive servo control[14] and the inverse kinematics by the Newton method. The force controller is applied as the upper layer of the position controller[15]. (See Fig. 2)

Then the posture control system is applied as a supervisory control to the reactive force controller. The objective is to make the center of mass (COM) of the robot and the

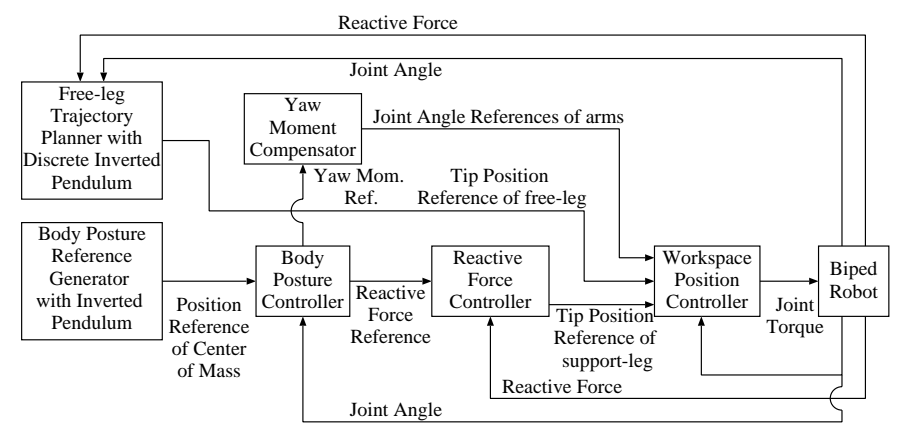

Fig. 2. Biped walking control system.

attitude of the body converge to its given reference trajectories. The parallel motion of the COM of the robot and the rotational motion of the body can be modeled from the first 6 rows of (7) and (8) as follows.

$$
\begin{aligned}
\tilde{\boldsymbol{H}} \dot{\tilde{\boldsymbol{v}}}+\tilde{\boldsymbol{b}} & =\tilde{\boldsymbol{u}}_{E} \\
\tilde{\boldsymbol{u}}_{E} & =\tilde{\boldsymbol{K}} \boldsymbol{f}_{A}
\end{aligned}
$$

and

$$
\begin{aligned}
\tilde{\boldsymbol{x}}^{T} & =\left[\begin{array}{ll}
\boldsymbol{p}_{C}^{T} & \boldsymbol{A}_{B}^{T}
\end{array}\right] \\
\tilde{\boldsymbol{v}}^{T} & =\left[\begin{array}{lll}
\boldsymbol{v}_{C}^{T} & \boldsymbol{\omega}_{B}^{T}
\end{array}\right] \\
\boldsymbol{f}_{A}^{T} & =\left[\begin{array}{llll}
\boldsymbol{f}_{R}^{T} & \boldsymbol{n}_{R}^{T} & \boldsymbol{f}_{L}^{T} & \boldsymbol{n}_{L}^{T}
\end{array}\right] \\
\tilde{\boldsymbol{K}} & =\left[\begin{array}{cccc}
\boldsymbol{I}_{3} & \mathbf{0} & \boldsymbol{I}_{3} & \mathbf{0} \\
{\left[\begin{array}{llll}
\boldsymbol{x}_{R} \times
\end{array}\right]} & \boldsymbol{I}_{3} & {\left[\boldsymbol{x}_{L} \times\right]} & \boldsymbol{I}_{3}
\end{array}\right] \\
\dot{\boldsymbol{p}}_{C} & =\boldsymbol{v}_{C}
\end{aligned}
$$

where

$\tilde{\boldsymbol{H}}: 6 \times 6$ inertia matrix

$\tilde{\boldsymbol{b}}: 6 \times 1$ vector specifying gravity effect and non-linear terms

$\tilde{\boldsymbol{u}}_{E}: 6 \times 1$ vector specifying external force at COM and external torque around body

$\boldsymbol{p}_{C}: 3 \times 1$ vector specifying $\mathrm{COM}$ of the robot

The ideal external force input $\tilde{\boldsymbol{u}}_{E}^{*}$ at the COM of the robot and around the body is determined by the state feedback.

$$
\tilde{\boldsymbol{u}}_{E}^{*}=\tilde{\boldsymbol{H}}_{n}\left[\boldsymbol{K}_{p}\left(\tilde{\boldsymbol{x}}_{r e f}-\tilde{\boldsymbol{x}}\right)+\boldsymbol{K}_{d}\left(\dot{\tilde{\boldsymbol{x}}}_{r e f}-\dot{\tilde{\boldsymbol{x}}}\right)+\ddot{\tilde{\boldsymbol{x}}}_{r e f}\right]+\tilde{\boldsymbol{b}}_{n}
$$

where

$$
\begin{aligned}
\tilde{\boldsymbol{H}}_{n}: & 6 \times 6 \text { diagonal matrix specifying nominal inertia } \\
\tilde{\boldsymbol{b}}_{n}: & 6 \times 1 \text { constant vector specifying non-linear terms, } \\
& \text { gravity effect, and non-diagonal inertial force }
\end{aligned}
$$

and $\boldsymbol{K}_{p}, \boldsymbol{K}_{d}$ represent gain matrices. The posture reference $\tilde{\boldsymbol{x}}_{r e f}$ is generated by linearized inverted pendulum models[16].

The contact force and torque are, however, physically limited to the repulsive condition, the friction condition, and zero moment point (ZMP) condition. Thus the ideal force $\tilde{\boldsymbol{u}}_{E}^{*}$ is not always realized by the reactive force/torque 
$\boldsymbol{f}_{A}$. We need to consider the following physical conditions. Let $\boldsymbol{f}_{R}^{T}=\left[\begin{array}{lll}f_{R x} & f_{R y} & f_{R z}\end{array}\right], \boldsymbol{n}_{R}^{T}=\left[\begin{array}{lll}n_{R x} & n_{R y} & n_{R z}\end{array}\right]$, $\boldsymbol{f}_{L}^{T}=\left[\begin{array}{lll}f_{L x} & f_{L y} & f_{L z}\end{array}\right]$, and $\boldsymbol{n}_{L}^{T}=\left[\begin{array}{ll}n_{L x} & n_{L y} \\ n_{L z}\end{array}\right]$. The normal component of the reactive force on the ground plane is not attractive but repulsive, which yields the following non-negative conditions.

$$
f_{R z} \geq 0, \quad f_{L z} \geq 0
$$

The friction force, i. e., the tangent component of the reactive force on the ground plane always exists within the friction cone.

$$
\begin{array}{cl}
\sqrt{f_{R x}^{2}+f_{R y}^{2}} \leq \mu f_{R z} & \sqrt{f_{L x}^{2}+f_{L y}^{2}} \leq \mu f_{L z} \\
\left|n_{R z}\right| \leq \mu^{\prime} f_{R z} & \left|n_{L z}\right| \leq \mu^{\prime} f_{L z}
\end{array}
$$

where $\mu$ and $\mu^{\prime}$ denote friction coefficients. It is possible to break out slips at the contact points when the equality in the (18)-(19) is realized.

The tangent component of the reactive torque at the center of the foot on the ground plane is also limited due to finiteness of the contact area.

$$
\begin{array}{ll}
\left|n_{R x}\right| \leq d_{y} f_{R z} & \left|n_{L x}\right| \leq d_{y} f_{L z} \\
\left|n_{R y}\right| \leq d_{x} f_{R z} & \left|n_{L y}\right| \leq d_{x} f_{L z}
\end{array}
$$

where $d_{x}$ and $d_{y}$ denote a half of the length and the width of the foot, respectively. (20)-(21) are equivalent to the zero moment point conditions.

Due to the physical limitations (17)-(21), the ideal force input $\tilde{\boldsymbol{u}}_{E}^{*}$ by (16) cannot be always realized by the reactive force and torque $\boldsymbol{f}_{A}$. Thus the following performance indices are introduced, which should be minimized under the limitations.

$$
\begin{aligned}
J_{\text {main }} & =\frac{1}{2}\left(\boldsymbol{u}_{E}-\boldsymbol{u}_{E}^{*}\right)^{T} \boldsymbol{C}_{1}\left(\boldsymbol{u}_{E}-\boldsymbol{u}_{E}^{*}\right) \\
J_{\text {sub }} & =\frac{1}{2} \boldsymbol{u}_{E}^{T} \boldsymbol{C}_{2} \boldsymbol{u}_{E}
\end{aligned}
$$

The index $J_{\text {main }}$ corresponds to the square error between the ideal force/torque and the realizable ones. The index $J_{\text {sub }}$ corresponds to the square error between the force and torque of the left foot and those of the right one. The reactive force and torque input $\boldsymbol{f}_{A}$ is determined by the quadratic programming, which minimizes the performance index under the linearized constraints of (17)-(21).

$$
\min _{f_{A}} J_{\text {main }}+\epsilon J_{\text {sub }}
$$

subject to $\quad \boldsymbol{A} \boldsymbol{f}_{A} \leq \boldsymbol{b}$

where

$$
\begin{aligned}
\boldsymbol{C}_{1} & =\operatorname{diag}\left\{w_{1}, w_{2}, w_{3}, w_{4}, w_{5}, w_{6}\right\} \\
\boldsymbol{C}_{2} & =\left[\begin{array}{cc}
\boldsymbol{I}_{6} & -\boldsymbol{I}_{6} \\
-\boldsymbol{I}_{6} & \boldsymbol{I}_{6}
\end{array}\right] \\
\boldsymbol{A} & =\left[\begin{array}{cc}
\boldsymbol{A}^{\prime} & \mathbf{0} \\
\mathbf{0} & \boldsymbol{A}^{\prime}
\end{array}\right]
\end{aligned}
$$

$$
\begin{aligned}
& \boldsymbol{A}^{\prime}=\left[\begin{array}{cccccc}
1 & 0 & -\mu & 0 & 0 & 0 \\
-1 & 0 & -\mu & 0 & 0 & 0 \\
0 & 1 & -\mu & 0 & 0 & 0 \\
0 & -1 & -\mu & 0 & 0 & 0 \\
0 & 0 & 1 & 0 & 0 & 0 \\
0 & 0 & -1 & 0 & 0 & 0 \\
0 & 0 & -d_{y} & 1 & 0 & 0 \\
0 & 0 & -d_{y} & -1 & 0 & 0 \\
0 & 0 & -d_{x} & 0 & 1 & 0 \\
0 & 0 & -d_{x} & 0 & -1 & 0 \\
0 & 0 & -\mu^{\prime} & 0 & 0 & 1 \\
0 & 0 & -\mu^{\prime} & 0 & 0 & -1
\end{array}\right] \\
& \boldsymbol{b}^{T}=\left[\begin{array}{ll}
\boldsymbol{b}_{R}^{T} & \boldsymbol{b}_{L}^{T}
\end{array}\right] \\
& \boldsymbol{b}_{R}^{T}=\left[\begin{array}{llllllllllll}
0 & 0 & 0 & 0 & z_{R} & 0 & 0 & 0 & 0 & 0 & 0 & 0
\end{array}\right] \\
& \boldsymbol{b}_{L}^{T}=\left[\begin{array}{llllllllllll}
0 & 0 & 0 & 0 & z_{L} & 0 & 0 & 0 & 0 & 0 & 0 & 0
\end{array}\right] \\
& z_{R}=\left\{\begin{array}{cl}
\text { any } & \text { if right leg is in support phase } \\
0 & \text { otherwise }
\end{array}\right. \\
& z_{L}=\left\{\begin{array}{cl}
\text { any } & \text { if left leg is in support phase } \\
0 & \text { otherwise }
\end{array}\right.
\end{aligned}
$$

and $\epsilon$ is a small positive real number.

The main performance index $J_{\text {main }}$ approaches the solution to the ideal force and torque $\boldsymbol{u}_{c}^{*}$ given by the state feedback. The sub performance index $J_{\text {sub }}$ distributes the inner force and torque to the both foot in balance. Because $\epsilon$ is very small, the sub performance index has almost no influence on the main performance index. The constrained (25) acts as a kind of a limiter in multi-input systems.

The optimization problem (24) and (25) are equivalent to the following quadratic programming problem.

$$
\begin{aligned}
\min _{f_{A}} & \frac{1}{2} \boldsymbol{f}_{A}^{T} \boldsymbol{Q} \boldsymbol{f}_{A}-\boldsymbol{c}_{0}^{T} \boldsymbol{f}_{A} \\
\text { subject to } & \boldsymbol{A} \boldsymbol{f}_{A} \leq \boldsymbol{b}
\end{aligned}
$$

where $\boldsymbol{Q}=\tilde{\boldsymbol{K}}^{T} \boldsymbol{C}_{1} \tilde{\boldsymbol{K}}+\epsilon \boldsymbol{C}_{2}$ and $\boldsymbol{c}_{0}=\tilde{\boldsymbol{K}}^{T} \boldsymbol{C}_{1} \boldsymbol{u}_{c}^{*}$. The reactive force and torque reference can be obtained by solving it at each sampling period.

\section{B. Free-leg Trajectory Planner}

Owing to the posture controller with the environmental interaction mentioned above, the on-line type autonomous walking control system can be realized as follows.

The posture controller mentioned above stabilizes the robot in the single and double support phases but does not realize the global locomotion. In order to achieve a stable walking, the foot must be periodically landed at adequate point. In this section, the tracking control of the COM by planning the land point of the foot is described.

The dynamics of the COM of the robot behaves like an inverted pendulum with the posture controller, i. e., $\ddot{p}_{c x}=$ $\omega^{2}\left(p_{c x}-p_{g x}\right)$ in sagittal plane and $\ddot{p}_{c y}=\omega^{2}\left(p_{c y}-p_{g y}\right)$ in lateral plane, where $p_{c x}$ an $p_{c y}$ denote the COM position in sagittal plane and in lateral plane, respectively. $p_{g x}$ and $p_{g y}$ denote the foot position of the support leg with respect to the origin of the world fixed coordinates in sagittal plane and in lateral plane, respectively. Digitalizing the inverted 
pendulum with one step period $T$, we have the discretetime inverted pendulum (37). (Here, only the equations in the saggital plane are described. The forms of the equations in the lateral plane are completely the same.)

$\left[\begin{array}{c}p_{c x}(t+T) \\ \dot{p}_{c x}(t+T)\end{array}\right]=\left[\begin{array}{cc}c & \frac{s}{\omega} \\ \omega s & c\end{array}\right]\left[\begin{array}{c}p_{c x}(t) \\ \dot{p}_{c x}(t)\end{array}\right]+\left[\begin{array}{c}1-c \\ -\omega s\end{array}\right] p_{g x}(t)$

where $c=\cosh \omega T$ and $s=\sinh \omega T$.

The movement of the free-leg must just begin at one step period in advance. Thus the augmented system including one step period delay is considered

$$
\begin{aligned}
& {\left[\begin{array}{l}
p_{c x}(t+T) \\
\dot{p}_{c x}(t+T) \\
p_{g x}(t+T)
\end{array}\right]=} \\
& {\left[\begin{array}{ccc}
c & s / \omega & 1-c \\
\omega s & c & -\omega s \\
0 & 0 & 0
\end{array}\right]\left[\begin{array}{c}
p_{c x}(t) \\
\dot{p}_{c x}(t) \\
p_{g x}(t)
\end{array}\right]+\left[\begin{array}{l}
0 \\
0 \\
1
\end{array}\right] p_{g x}^{r e f}(t)}
\end{aligned}
$$

The reference of the landing point $p_{g x}^{r e f}(t)$ which sets the system characteristic polynomial $\phi(z)$ to $\phi(z)=z^{3}+\alpha_{2} z^{2}+$ $\alpha_{1} z+\alpha_{0}$ is obtained by the state feedback as follows.

$$
\begin{aligned}
& p_{g x}^{r e f}(t+ \\
& \quad \\
& \quad f_{0}\left[p_{c x}(t)-p_{c x}^{c m d}(t)\right]+f_{1}\left[\dot{p}_{c x}(t)-\dot{p}_{c x}^{c m d}(t)\right] \\
& \quad+f_{2}\left[p_{g x}(t)-p_{g x}^{c m d}(t)\right]+p_{g x}^{c m d}(t+T)
\end{aligned}
$$

where

$$
\begin{aligned}
f_{0} & =\frac{1-\alpha_{0}-\alpha_{1}+\alpha_{2}+2\left(1-\alpha_{2}\right) c-4 c^{2}}{2(c-1)} \\
f_{1} & =\frac{1+\alpha_{0}-\alpha_{1}-\alpha_{2}-2\left(1+\alpha_{2}\right) c-4 c^{2}}{2 \omega s} \\
f_{2} & =\alpha_{2}+2 c
\end{aligned}
$$

$p_{c x}^{c m d}$ denotes the reference trajectory of COM in sagittal plane and $p_{g x}^{c m d}$ denotes the offset of the landing points synchronizing trajectory of COM in sagittal plane.

The trajectory of the free-leg is given by connecting the next and previous landing point with a smooth function as follows.

$$
\begin{aligned}
p_{g x}^{r e f}(t+ & m)=p_{g x}^{r e f}(t-T) \\
& +\frac{1-\cos \pi m}{2}\left[p_{g x}^{r e f}(t+T)-p_{g x}^{r e f}(t-T)\right]
\end{aligned}
$$

and the height of free-leg is also given by

$$
p_{g z}^{r e f}(t+m)=h^{r e f} \frac{1-\cos 2 \pi m}{2}
$$

where $0 \leq m \leq T$.

The global system configuration is shown in Fig. 2.

\section{New Simulation Scheme}

This section describes a new simulation method of multi-degree-of-freedom mechanical systems with the timedependent contact and the Coulomb friction, such as a legged robot interacting with a ground, a satellite-mounted manipulator catching an object, etc. The proposed method is the extension of the open link manipulator simulation[20] and the contact simulation of rigid body mechanics[21]. The proposed simulation model is mathematically exact, thus this simulator enables the essential investigation for control algorithms of the mechanical systems.

\section{A. Numerical Integration}

It is easy to simulate the dynamic motion of a legged robot (4)-(7) numerically by integrating $\dot{\boldsymbol{v}}$ and $\boldsymbol{v}$ on each time step after solving (7) for the acceleration $\dot{\boldsymbol{v}}$ given $\boldsymbol{x}$, $\boldsymbol{v}, \boldsymbol{u}$, and $\boldsymbol{u}_{E}$. In a case of the Euler integration, we have

$$
\begin{aligned}
\boldsymbol{p}_{B}(t+h)= & \boldsymbol{p}_{B}(t)+h \boldsymbol{v}_{B}(t) \\
\boldsymbol{A}_{B}(t+h)= & \boldsymbol{T}\left(h \boldsymbol{\omega}_{B}(t)\right) \boldsymbol{A}_{B}(t) \\
\boldsymbol{\theta}(t+h)= & \boldsymbol{\theta}(t)+h \boldsymbol{\omega}(t) \\
\boldsymbol{v}(t+h)= & \boldsymbol{v}(t)+h \boldsymbol{H}(\boldsymbol{x}(t))^{-1}[\boldsymbol{u}(t) \\
& \left.+\boldsymbol{u}_{E}(\boldsymbol{x}(t), \boldsymbol{v}(t))-\boldsymbol{b}(\boldsymbol{x}(t), \boldsymbol{v}(t))\right]
\end{aligned}
$$

where biasing vector $\boldsymbol{b}(\boldsymbol{x}(t), \boldsymbol{v}(t))=\boldsymbol{C}(\boldsymbol{x}(t), \boldsymbol{v}(t)) \boldsymbol{v}(t)+$ $\boldsymbol{g}(\boldsymbol{x}(t))$, and $h$ is the time-step. $\boldsymbol{T}\left(h \boldsymbol{\omega}_{B}\right)$ acts as a rotational transformer around $\boldsymbol{\omega}_{B}$ axis with angle $h\left|\boldsymbol{\omega}_{B}\right|$. (See [18] and Fig. 3.)

$\boldsymbol{T}\left(h \boldsymbol{\omega}_{B}\right)=\left[(\cos \psi) \boldsymbol{I}_{3}+(1-\cos \psi) \boldsymbol{r} \boldsymbol{r}^{T}+(\sin \psi)[\boldsymbol{r} \times]\right]$

where $\psi=h\left|\boldsymbol{\omega}_{B}\right|, \boldsymbol{r}=\boldsymbol{\omega}_{B} /\left|\boldsymbol{\omega}_{B}\right|$.

$\boldsymbol{H}(\boldsymbol{x})$ and $\boldsymbol{b}(\boldsymbol{x}, \boldsymbol{v})$ can be obtained by the inverse dynamics calculation using the Newton-Euler formulation, $i$. e., given $\boldsymbol{x}, \boldsymbol{v}$, and $\boldsymbol{v}$ solve for $\boldsymbol{u}$. In fact, $\boldsymbol{H}(\boldsymbol{x})$ can be calculated by solving inverse dynamics with setting $\boldsymbol{x}$ to the current state, $\boldsymbol{v}=\boldsymbol{e}_{j}$, and ignoring centrifugal forces, Corioli's forces, gravity effects, and external forces[20]. Here, $\boldsymbol{e}_{j}$ means a unit vector with its $j$ th element equal to 1 and others are 0 . The solution about $\boldsymbol{u}$ corresponds to the $j$ th column of $\boldsymbol{H}$. (See (7).) The biasing vector $\boldsymbol{b}(\boldsymbol{x}, \boldsymbol{v})$ can be also computed by setting $\dot{\boldsymbol{v}}=0$.

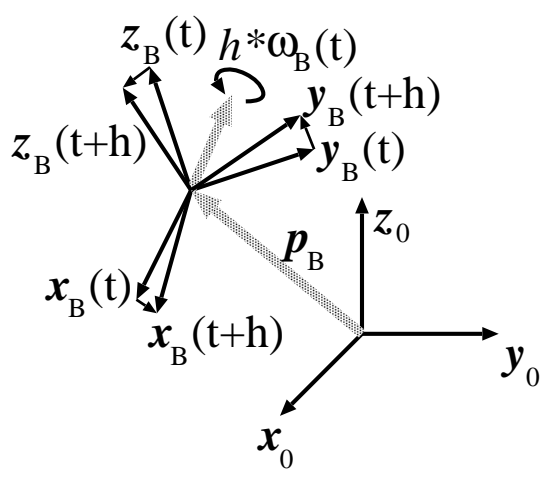

Fig. 3. Rotation of coordinates.

\section{B. Inverse Dynamics}

The inverse dynamics can be calculated by following recursive equations. The formulation is based on the algo- 


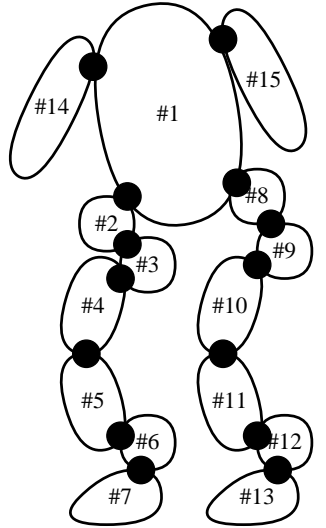

Fig. 4. Indices of links.

TABLE I

EXAMPles OF A INNER LINK AND OUTER LiNKS IN Fig. 4 CASE.

\begin{tabular}{cccccc}
\hline $\operatorname{link} i$ & 1 & 2 & 7 & 8 & 14 \\
\hline inner link $\underline{i}$ & - & 1 & 6 & 1 & 1 \\
outer links $O_{i}$ & $2,8,14,15$ & 3 & $\emptyset$ & 9 & $\emptyset$
\end{tabular}

rithm in [19], but several points are different. In the proposed method, 1) there is no fixed point in the robotic system and the base-link is movable, and 2) the expression of the link-fixed coordinates is modified to deal with branching links.

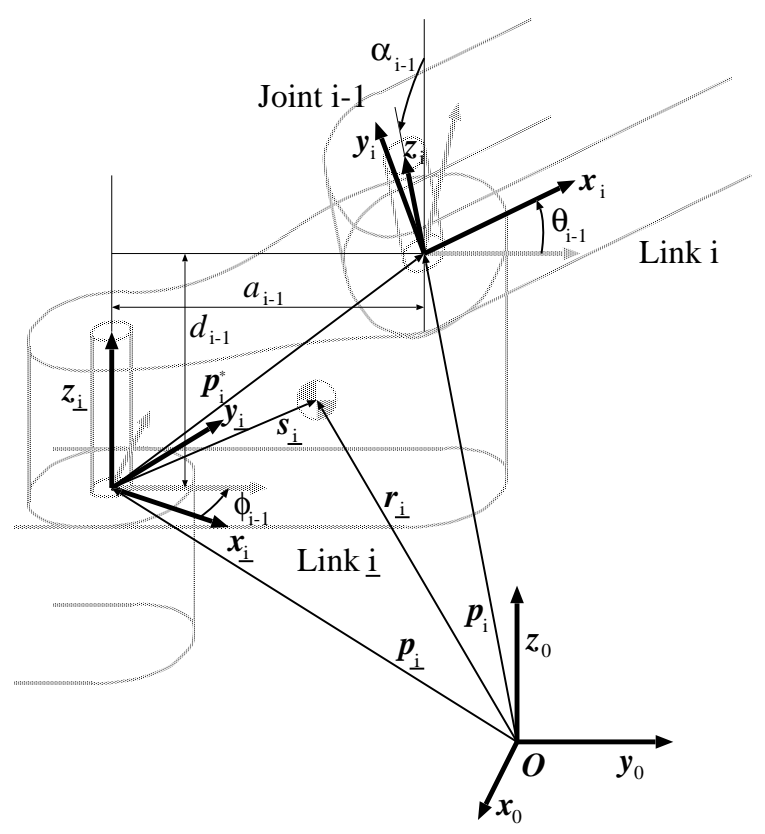

Fig. 5. Coordinates and parameters of links.

The index numbers of links are introduced as shown in Fig. 4 in a 14-axis biped case. Here, the link $\underline{i}$ is defined as the inner link of $i$ th link, whose index is the small- est in all links connected to $i$ th link. Also the set of links $O_{i}$ is defined as the outer links of $i$ th link, whose indices are larger than $i$ in all links connected to $i$ th link. The examples are shown in Table I. To compute the inverse dynamics efficiently, the link-fixed coordinates shown in Fig. 5 are introduced. The $i$ th coordinates are defined as ${ }^{0} \boldsymbol{A}_{i}={ }^{0} \boldsymbol{A}_{1}{ }^{1} \boldsymbol{A}_{2} \ldots{ }^{i} \boldsymbol{A}_{i}$ and ${ }^{0} \boldsymbol{A}_{1}=\boldsymbol{A}_{B}$, where ${ }^{i} \boldsymbol{A}_{i}=\left[{ }^{i} \boldsymbol{x}_{i},{ }_{\boldsymbol{y}_{i}}, \underline{\boldsymbol{z}}_{i}\right]$ corresponds to $i$ th coordinates referenced to $\underline{i}$ th coordinates system. ${ }^{i} \boldsymbol{A}_{i}$ transforms any vector with reference to $i$ th coordinates system to one in $\underline{i}$ th coordinates system. (Note: ${ }^{i} \boldsymbol{A}_{i}^{-1}={ }^{i} \boldsymbol{A}_{i}^{T}={ }^{i} \boldsymbol{A}_{\underline{i}}$ ). In a case of the parametrization of Fig. 5 ,

$$
{ }^{i} \boldsymbol{A}_{i}=\left[\begin{array}{ccc}
c_{\phi} c_{\theta}-s_{\phi} c_{\alpha} s_{\theta} & -c_{\phi} s_{\theta}-s_{\phi} c_{\alpha} c_{\theta} & s_{\phi} s_{\alpha} \\
s_{\phi} c_{\theta}+c_{\phi} c_{\alpha} s_{\theta} & -s_{\phi} s_{\theta}+c_{\phi} c_{\alpha} c_{\theta} & -c_{\phi} s_{\alpha} \\
s_{\alpha} s_{\theta} & s_{\alpha} c_{\theta} & c_{\alpha}
\end{array}\right]
$$

where $s_{\phi}=\sin \phi_{i-1}, c_{\phi}=\cos \phi_{i-1}, s_{\alpha}=\sin \alpha_{i-1}, c_{\alpha}=$ $\cos \alpha_{i-1}, s_{\theta}=\sin \theta_{i-1}$, and $c_{\theta}=\cos \theta_{i-1}$.

When $\dot{\boldsymbol{v}}_{B}, \dot{\boldsymbol{\omega}}_{B}, \boldsymbol{\omega}_{B}, \ddot{\theta}_{i}, \dot{\theta}_{i}$ and $\theta_{i}$ are given, the angular velocity ${ }^{i} \boldsymbol{\omega}_{i}$, the angular acceleration ${ }^{i} \dot{\boldsymbol{\omega}}_{i}$, and the acceleration of the origin ${ }^{i} \ddot{\boldsymbol{p}}_{i}$ of $i$ th link referenced to its own link coordinates can be recurrently obtained as follows. For $i=1$

$$
\begin{aligned}
{ }^{1} \ddot{\boldsymbol{p}}_{1} & ={ }^{1} \boldsymbol{A}_{0}\left(\dot{\boldsymbol{v}}_{B}+\boldsymbol{g}\right) \\
{ }^{1} \boldsymbol{\omega}_{1} & ={ }^{1} \boldsymbol{A}_{0} \boldsymbol{\omega}_{B} \\
{ }^{1} \dot{\boldsymbol{\omega}}_{1} & ={ }^{1} \boldsymbol{A}_{0} \dot{\boldsymbol{\omega}}_{B}
\end{aligned}
$$

For $2 \leq i \leq N$

$$
\begin{aligned}
{ }^{i} \boldsymbol{\omega}_{i} & ={ }^{i} \boldsymbol{A}_{\underline{i}}{ }^{i} \boldsymbol{\omega}_{\underline{i}}+\boldsymbol{z}_{0} \dot{\theta}_{i-1} \\
{ }^{i} \dot{\boldsymbol{\omega}}_{i} & ={ }^{i} \boldsymbol{A}_{\underline{i}}{ }^{i} \dot{\boldsymbol{\omega}}_{\underline{i}}+\boldsymbol{z}_{0} \ddot{\theta}_{i-1}+\left({ }^{i} \boldsymbol{A}_{\underline{i}} \boldsymbol{\omega}_{\underline{i}}\right) \times \boldsymbol{z}_{0} \dot{\theta}_{i-1} \\
{ }^{i} \ddot{\boldsymbol{p}}_{i} & ={ }^{i} \boldsymbol{A}_{\underline{i}}\left[{ }^{i} \dot{\boldsymbol{\omega}}_{\underline{i}} \times{ }^{i} \boldsymbol{p}_{i}^{*}+{ }^{i} \boldsymbol{\omega}_{\underline{i}} \times\left({ }^{i} \boldsymbol{\omega}_{\underline{i}} \times{ }^{i} \boldsymbol{p}_{i}^{*}\right)+{ }^{i} \ddot{\boldsymbol{p}}_{\underline{i}}\right]
\end{aligned}
$$

Thus the acceleration of the center of mass $\ddot{\boldsymbol{r}}_{i}$, the total force $\boldsymbol{F}_{i}$, and the total moment $\boldsymbol{N}_{i}$ of the $i$ th link can be calculated as follows. For $1 \leq i \leq N$

$$
\begin{aligned}
{ }^{i} \ddot{\boldsymbol{r}}_{i} & ={ }^{i} \dot{\boldsymbol{\omega}}_{i} \times{ }^{i} \boldsymbol{s}_{i}+{ }^{i} \boldsymbol{\omega}_{i} \times\left({ }^{i} \boldsymbol{\omega}_{i} \times{ }^{i} \boldsymbol{s}_{i}\right)+{ }^{i} \ddot{\boldsymbol{p}}_{i} \\
{ }^{i} \boldsymbol{F}_{i} & ={ }^{i} m_{i}{ }_{i} \ddot{\boldsymbol{r}}_{i} \\
{ }^{i} \boldsymbol{N}_{i} & ={ }^{i} \boldsymbol{J}_{i}{ }_{i} \dot{\boldsymbol{\omega}}_{i}+{ }^{i} \boldsymbol{\omega}_{i} \times\left({ }^{i} \boldsymbol{J}_{i}{ }^{i} \boldsymbol{\omega}_{i}\right)
\end{aligned}
$$

Here, $\boldsymbol{p}_{i}^{*}$ denotes $\boldsymbol{p}_{i}-\boldsymbol{p}_{\underline{i}}$ which is calculated as ${ }_{i}^{\boldsymbol{p}_{i}^{*}}=$ $\left[a_{i-1} \cos \phi_{i-1}, a_{i-1} \sin \phi_{i-1}, d_{i-1}\right]^{T} . \quad \boldsymbol{z}_{i}$ denotes the direction of the joint $i-1$, and $\boldsymbol{s}_{i}$ denotes the center of mass with respect to the origin of link $i$ coordinates. (See Fig. 5.) The gravity effect $\boldsymbol{g}$ is considered in (51).

Then, $\boldsymbol{f}_{i}$ and $\boldsymbol{n}_{i}$, the force and moment exerted on link $i$ by inner link $\underline{i}$ can be calculated as follows. For $1 \leq i \leq N$

$$
\begin{aligned}
{ }^{i} \boldsymbol{f}_{i}= & { }^{i} \boldsymbol{F}_{i}+\sum_{j \in O_{i}}{ }^{j} \boldsymbol{A}_{j}{ }^{j} \boldsymbol{f}_{j}+{ }^{i} \boldsymbol{A}_{0} \sum_{j \in M_{i}}{ }^{0} \boldsymbol{f}_{E j} \\
{ }^{i} \boldsymbol{n}_{i}= & { }^{i} \boldsymbol{N}_{i}+\sum_{j \in O_{i}}\left[{ }^{j} \boldsymbol{A}_{j}{ }^{j} \boldsymbol{n}_{j}+{ }^{j} \boldsymbol{p}_{j}^{*} \times\left({ }^{j} \boldsymbol{A}_{j}{ }^{j} \boldsymbol{f}_{j}\right)\right] \\
& +{ }^{i} \boldsymbol{s}_{i} \times{ }^{i} \boldsymbol{F}_{i}-\sum_{j \in M_{i}}\left[{ }^{j} \boldsymbol{c}_{j} \times\left({ }^{i} \boldsymbol{A}_{0}{ }^{0} \boldsymbol{f}_{E j}\right)\right]
\end{aligned}
$$


where $f_{E j}$ is the $j$ th external force. $M_{i}$ is a set of index numbers of external forces which are imposed on link $i . \boldsymbol{c}_{j}$ is the position vector of $j$ th contact point with respect to the origin of its own link-fixed coordinates as shown in Fig. 6

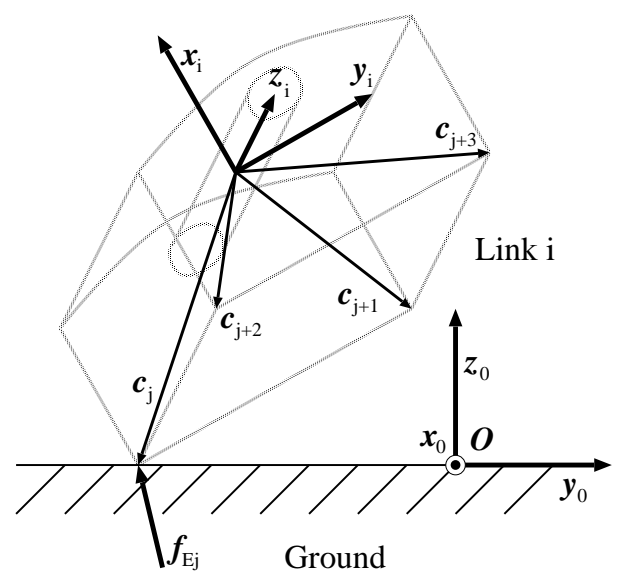

Fig. 6. Contact points.

As a result, the solution of the inverse dynamics $\boldsymbol{u}^{T}=$ $\left[\boldsymbol{f}_{B}^{T}, \boldsymbol{n}_{B}^{T}, \boldsymbol{\tau}^{T}\right]$ given $\boldsymbol{x}, \boldsymbol{v}$, and $\boldsymbol{v}$ is obtained as follows, where $\boldsymbol{f}_{B}$ and $\boldsymbol{n}_{B}$ are the force and the moment exerted on the origin of the base link, respectively, and $\tau_{i}$ is the torque on $i$ th joint. For $1 \leq i \leq N-1$

$$
\tau_{i}={ }^{i+1} \boldsymbol{n}_{i+1}^{T} \boldsymbol{z}_{0}-D_{i} \dot{\theta}_{i}
$$

For $i=1$

$$
\begin{aligned}
& \boldsymbol{f}_{B}={ }^{0} \boldsymbol{A}_{1}{ }^{1} \boldsymbol{f}_{1} \\
& \boldsymbol{n}_{B}={ }^{0} \boldsymbol{A}_{1}{ }^{1} \boldsymbol{n}_{1}
\end{aligned}
$$

where $D_{i}$ is the viscous damping coefficient of joint $i$.

\section{Constrained Force}

In order to support the biped robot on the ground, the appropriate reactive forces from the ground should be determined at each time step in the simulation. In general, springs and dampers model at the contact points are introduced as a collision models. That simulation model, however, requires more shorter time step than the mechanical system itself does. Therefore we introduce a collision model based on a mathematical programming, which is the extension of the plastic collision model[21] and can deal with the three dimensional contact and arbitrary repulsion between plasticity and elasticity. In addition, a motion with the continuous collision behaves an exact ideal constrained motion.

In general, the external force on the generalized coordinates $\boldsymbol{u}_{E}$ in (7) is expressed by all external forces on contact points as follows.

$\boldsymbol{u}_{E}=\sum_{i \in M_{A}} \boldsymbol{K}_{i} \boldsymbol{f}_{E i}=\left[\begin{array}{lll}\boldsymbol{K}_{x} & \boldsymbol{K}_{y} & \boldsymbol{K}_{z}\end{array}\right]\left[\begin{array}{l}\boldsymbol{f}_{E x} \\ \boldsymbol{f}_{E y} \\ \boldsymbol{f}_{E z}\end{array}\right]=\boldsymbol{K} \boldsymbol{f}_{E}$ where $M_{A}$ represents a set of index numbers of active contact points. $\boldsymbol{f}_{E x}$ and $\boldsymbol{f}_{E y}$ consist of tangent components of all active contact forces. Also $\boldsymbol{f}_{E z}$ consists of normal components of all active contact forces. $\boldsymbol{K}_{x}, \boldsymbol{K}_{y}$, and $\boldsymbol{K}_{z}$ correspond to those transformers. The dimensions of $\boldsymbol{K}$ and $f_{E}$ are depend on the number of the contact points which is time-variant. In advance, $\boldsymbol{K}$ can be obtained by solving the inverse dynamics mentioned above with setting $\boldsymbol{x}$ to the current state, $\boldsymbol{f}_{E}=\boldsymbol{e}_{j}, \boldsymbol{v}=0$, and ignoring gravity, centrifugal, and Corioli's effects.

When the external impulsive force $\Delta f\left(=f_{E} \Delta t\right)$ are imposed in the system (7), the following equation is realized.

$$
\boldsymbol{H}\left(\boldsymbol{v}_{+}-\boldsymbol{v}_{-}\right)=\Delta \boldsymbol{f}_{b}+\boldsymbol{K} \Delta \boldsymbol{f}
$$

where $\boldsymbol{v}_{+}$and $\boldsymbol{v}_{-}$denote the velocity after the collision and before the collision, respectively. $\Delta \boldsymbol{f}_{b}$ denotes the uncontrollable impulse force, i. e., $\Delta \boldsymbol{f}_{b} \approx\left[\boldsymbol{u}-\boldsymbol{C}\left(\boldsymbol{x}, \boldsymbol{v}_{-}\right) \boldsymbol{v}_{-}-\right.$ $\boldsymbol{g}(\boldsymbol{x})] \Delta t$. Thus, the kinetic energy after the collision can be calculated as follows,

$$
\begin{aligned}
& \frac{1}{2} \boldsymbol{v}_{+}^{T} \boldsymbol{H} \boldsymbol{v}_{+}=\frac{1}{2} \boldsymbol{v}_{-}^{T} \boldsymbol{H} \boldsymbol{v}_{-}+\frac{1}{2} \Delta \boldsymbol{f}^{T} \boldsymbol{K}^{T} \boldsymbol{H}^{-1} \boldsymbol{K} \Delta \boldsymbol{f} \\
&+\left(\boldsymbol{v}_{-}+\boldsymbol{H}^{-1} \Delta \boldsymbol{f}_{b}\right)^{T} \boldsymbol{K} \Delta \boldsymbol{f}+\frac{1}{2} \Delta \boldsymbol{f}_{b}^{T} \boldsymbol{H}^{-1} \Delta \boldsymbol{f}_{b}
\end{aligned}
$$

The plastic collision is defined as the energy minimization with given conditions[21] which yield a quadratic programming (QP) problem. In this paper, the model is expanded to the following problem to deal with three dimensional contact and arbitrary repulsion between plasticity and elasticity.

$$
\begin{array}{cl}
\underset{\Delta \boldsymbol{f}}{\operatorname{minimize}} & \frac{1}{2} \Delta \boldsymbol{f}^{T} \boldsymbol{Q}_{1} \Delta \boldsymbol{f}+\boldsymbol{q}_{1}^{T} \Delta \boldsymbol{f} \\
\text { subject to } & \Delta f_{z i} \geq 0 \text { and } \sqrt{\Delta f_{x i}^{2}+\Delta f_{y i}^{2}} \leq \mu \Delta f_{z i}
\end{array}
$$

where

$$
\begin{aligned}
\boldsymbol{Q}_{1} & =\boldsymbol{K}^{T} \boldsymbol{H}^{-1} \boldsymbol{K} \\
\boldsymbol{q}_{1} & =\boldsymbol{K}^{T}\left(\boldsymbol{v}_{-}+\boldsymbol{H}^{-1} \Delta \boldsymbol{f}_{b}\right)+\left[\begin{array}{c}
\mathbf{0} \\
\mathbf{0} \\
\lambda \boldsymbol{K}_{z}^{T}
\end{array}\right] \boldsymbol{v}_{-}
\end{aligned}
$$

And $\lambda$ represents a repulsion coefficient which is arbitrary set within $0 \leq \lambda \leq 1$.

The problem is equivalent to

minimize the optimal value of sub-problem (72), (73)

$\Delta \boldsymbol{f}_{\theta}$

$\underset{\Delta \boldsymbol{f}_{r}, \Delta \boldsymbol{f}_{z}}{\operatorname{minimize}} \quad \frac{1}{2}\left[\begin{array}{c}\Delta \boldsymbol{f}_{r} \\ \Delta \boldsymbol{f}_{z}\end{array}\right]^{T} \boldsymbol{Q}_{2}\left[\begin{array}{c}\Delta \boldsymbol{f}_{r} \\ \Delta \boldsymbol{f}_{z}\end{array}\right]+\boldsymbol{q}_{2}^{T}\left[\begin{array}{c}\Delta \boldsymbol{f}_{r} \\ \Delta \boldsymbol{f}_{z}\end{array}\right]$

subject to $\quad \Delta f_{z i} \geq 0$ and $\left|\Delta f_{r i}\right| \leq \mu \Delta f_{z i}$

where

$$
\boldsymbol{Q}_{2}=\left[\begin{array}{c}
\boldsymbol{K}_{r}^{T} \\
\boldsymbol{K}_{z}^{T}
\end{array}\right] \boldsymbol{H}^{-1}\left[\begin{array}{ll}
\boldsymbol{K}_{r} & \boldsymbol{K}_{z}
\end{array}\right]
$$




$$
\begin{aligned}
\boldsymbol{q}_{2} & =\left[\begin{array}{c}
\boldsymbol{K}_{r}^{T} \\
\boldsymbol{K}_{z}^{T}
\end{array}\right]\left(\boldsymbol{v}_{-}+\boldsymbol{H}^{-1} \Delta \boldsymbol{f}_{b}\right)+\left[\begin{array}{c}
\mathbf{0} \\
\lambda \boldsymbol{K}_{z}^{T}
\end{array}\right] \boldsymbol{v} \\
\Delta f_{r i} & =\sqrt{\Delta f_{x i}^{2}+\Delta f_{y i}^{2}} \\
\Delta f_{\theta i} & =\arctan \Delta f_{y i} / \Delta f_{x i} \\
\boldsymbol{K}_{r} & =\boldsymbol{K}_{x} \cos \Delta \boldsymbol{f}_{\theta}+\boldsymbol{K}_{y} \sin \Delta \boldsymbol{f}_{\theta}
\end{aligned}
$$

The constraints of the sub-problem become linear, so that the problem can be solved by non-constraint optimization algorithm (for example quasi Newton method), including the QP sub-problem.

The solution of the problem satisfy Kuhn-Tucker's necessary and sufficient conditions as follows.

$$
\begin{aligned}
& \mu \Delta f_{r i}^{*} \geq\left|\Delta f_{z i}^{*}\right| \\
& \Delta f_{r i}^{*} \boldsymbol{k}_{r i}^{T} \boldsymbol{v}_{+} \leq 0 \\
& \left(\mu \Delta f_{z i}^{*}-\left|\Delta f_{r i}^{*}\right|\right) \boldsymbol{k}_{r i}^{T} \boldsymbol{v}_{+}=0 \\
& \Delta f_{z i}^{*} \geq 0 \\
& \boldsymbol{k}_{z i}^{T} \boldsymbol{v}_{+}+\lambda \boldsymbol{k}_{z i}^{T} \boldsymbol{v}_{-} \geq 0 \\
& \begin{cases}\Delta f_{z i}^{*}\left(\boldsymbol{k}_{z i}^{T} \boldsymbol{v}_{+}+\lambda \boldsymbol{k}_{z i}^{T} \boldsymbol{v}_{-}\right)=0 & \text { if }\left|\Delta f_{r i}^{*}\right|<\mu \Delta f_{z i}^{*} \\
\boldsymbol{k}_{z i}^{T} \boldsymbol{v}_{+}+\lambda \boldsymbol{k}_{z i}^{T} \boldsymbol{v}_{-} \geq \mu\left|\boldsymbol{k}_{r i}^{T} \boldsymbol{v}_{+}\right| & \text {if }\left|\Delta f_{r i}^{*}\right|=\mu \Delta f_{z i}^{*}\end{cases}
\end{aligned}
$$

where $\boldsymbol{k}_{r i}$ and $\boldsymbol{k}_{z i}$ denote $i$ th row of the matrices $\boldsymbol{K}_{r}$ and $\boldsymbol{K}_{z}$, respectively.

Conditions (79) to (81) are related to the tangent motion. Here $\boldsymbol{k}_{r i}^{T} \boldsymbol{v}_{+}$corresponds to the tangent velocity after the collision due to the principle of the virtual work. Especially, (81) means that the friction condition is activated $\left(\left|\Delta f_{r i}^{*}\right|=\right.$ $\mu \Delta f_{z i}^{*}$ ) if the contact point slips. If the friction condition is not activated $\left(\left|\Delta f_{r i}^{*}\right|<\mu \Delta f_{z i}^{*}\right)$, the tangent velocity at the contact point becomes zero. (83) means that the method realizes arbitrary repulsion between plasticity and elasticity. The motion with the proposed collision behaves an exact ideal constrained motion.

\section{TABLE II}

PARAMETERS OF BIPED ROBOT.

\begin{tabular}{ccc}
\hline parts & size $[\mathrm{m}]$ & weight $[\mathrm{kg}]$ \\
\hline \hline all & 0.99 & 28.744 \\
\hline head & $0.14 \times 0.14 \times 0.14(d \times w \times h)$ & 2.744 \\
arm & 0.3 & 3.5 \\
body & 0.4 & 8 \\
thigh & 0.2 & 2 \\
shin & 0.2 & 2 \\
foot & $0.2 \times 0.1(d \times w)$ & 1.5 \\
\hline
\end{tabular}

\section{Simulation Results}

The proposed control is applied to 20 axes human-type biped robot and is investigated by the proposed precise $3 \mathrm{D}$ dynamic simulator. The parameters of the robot is shown in Table II. The control parameter is set as follows. The walking velocity: $0.25[\mathrm{~m} / \mathrm{s}]$, the walking period: 0.5 [sec/step], the double support period: 0.05 [sec], and the characteristic polynomial: $(z-0.3)^{3}$.

The QP is solved by the algorithm in [22].
The snapshots of the simulation is shown in Fig. 7. The initial movement of COM is finished between $[0,1]$. After that, the walking motion starts. Fig. 8 shows the trajectory of zero moment point (ZMP). Fig. 9 shows the response of $\mathrm{COM}$ with the reference trajectory, in which the tracking error converges to zero. From the magnified figures (c) and (d), the COM follows the inverted pendulum model even among the stepping period.

The walking motion becomes more robust when the yaw axis moment is compensated by the arm swing motion[17].

\section{Conclusions}

In this paper the following hierarchical control system is proposed. 1) a posture controller which consider the physical constraints of the reactive force/torque on the foot by quadratic programming. 2) a real-time COM tracking controller by the foot placement with a discrete inverted pendulum model. 3) a three dimensional dynamic simulation scheme with precise contact with the environment.

When The proposed control system is applied to the 20 axes simulation model, the stable biped locomotion with a velocity $0.25 \mathrm{~m} / \mathrm{sec}$ and a stepping time $0.5 \mathrm{sec} / \mathrm{step}$ is realized.

\section{REFERENCES}

[1] S. Kajita, T. Yamaura, and A. Kobayashi, "Dynamic Walking Control of a Biped Robot Along a Potential Energy Conserving Orbit," IEEE Trans. on Robotics and Automation, vol. 8, no. 4, pp. 431-438, 1992.

[2] J. Furusho and A. Sano, "Sensor-Based Control of a Nine-Link Biped," Int. J. Robotics Research, vol. 9, no. 2, pp. 83-98, 1990.

[3] J. Yamaguchi, A. Takanishi and I. Kato, "Development of a Biped Walking Robot Compensating for Three-Axis Moment by Trunk Motion," J. Robotics Society of Japan, vol. 11, no. 4, pp.581-586, 1993. (in Japanese)

[4] M. H. Raibert, Legged Robots That Balance, Cambridge, MA, MIT Press, 1986.

[5] A. Kun and W. T. Miller, III, "Adaptive Dynamic Balance of a Biped Robot using Neural Networks," Proc. IEEE Int. Conf. on Robotics and Automation, pp. 240-245, 1996.

[6] J. K. Hodgins, "Three-Dimensional Human Running," Proc. IEEE Int. Conf. on Robotics and Automation, pp. 3271-3276, 1996.

[7] J. Yamaguchi, N. Kinoshita, A. Takanishi, and I. Kato, "Development of a Dynamic Biped Walking System for Humanoid, Development of a Biped Walking Robot Adapting to the $\mathrm{Hu}-$ man's Living Floor -," Proc. IEEE Int. Conf. on Robotics and Automation, pp. 232-239, 1996.

[8] S. Kajita and K. Tani, "Adaptive Gait Control of a Biped Robot based on Realtime Sensing of the Ground Profile," Proc. IEEE Int. Conf. on Robotics and Automation, pp. 570-577, 1996.

[9] H. Minakata and Y. Hori, "Development of Biped Bike Prototype 'Ostrich-I\&II'," Proc. Asian Control Conference, vol. 3, pp. 319-322, 1997.

[10] A. W. Salatian, K. Y. Yi, and Y. F. Zheng, "Reinforcement Learning for a Biped Robot to Climb Sloping Surfaces," $J$. Robotic Systems, vol. 14, no. 4, pp. 283-296, 1997.

[11] T. Fukuda, Y. Komata, and T. Arakawa, "Stabilization Control of Biped Locomotion Robot based Learning with GAs having Self-adaptive Mutation and Recurrent Neural Networks," Proc. IEEE Int. Conf. on Robotics and Automation, pp. 217-222, 1997.

[12] J. Pratt, P. Dilworth, and G. Pratt, "Virtual Model Control of a Bipedal Walking Robot," Proc. IEEE Int. Conf. on Robotics and Automation, pp. 193-198, 1997.

[13] Y. Fujimoto and A. Kawamura, "Three Dimensional Digital Simulation and Autonomous Walking Control for Eight-Axis Biped Robot," Proc. IEEE Int. Conf. on Robotics and Automation, pp. 2877-2884, 1995. 


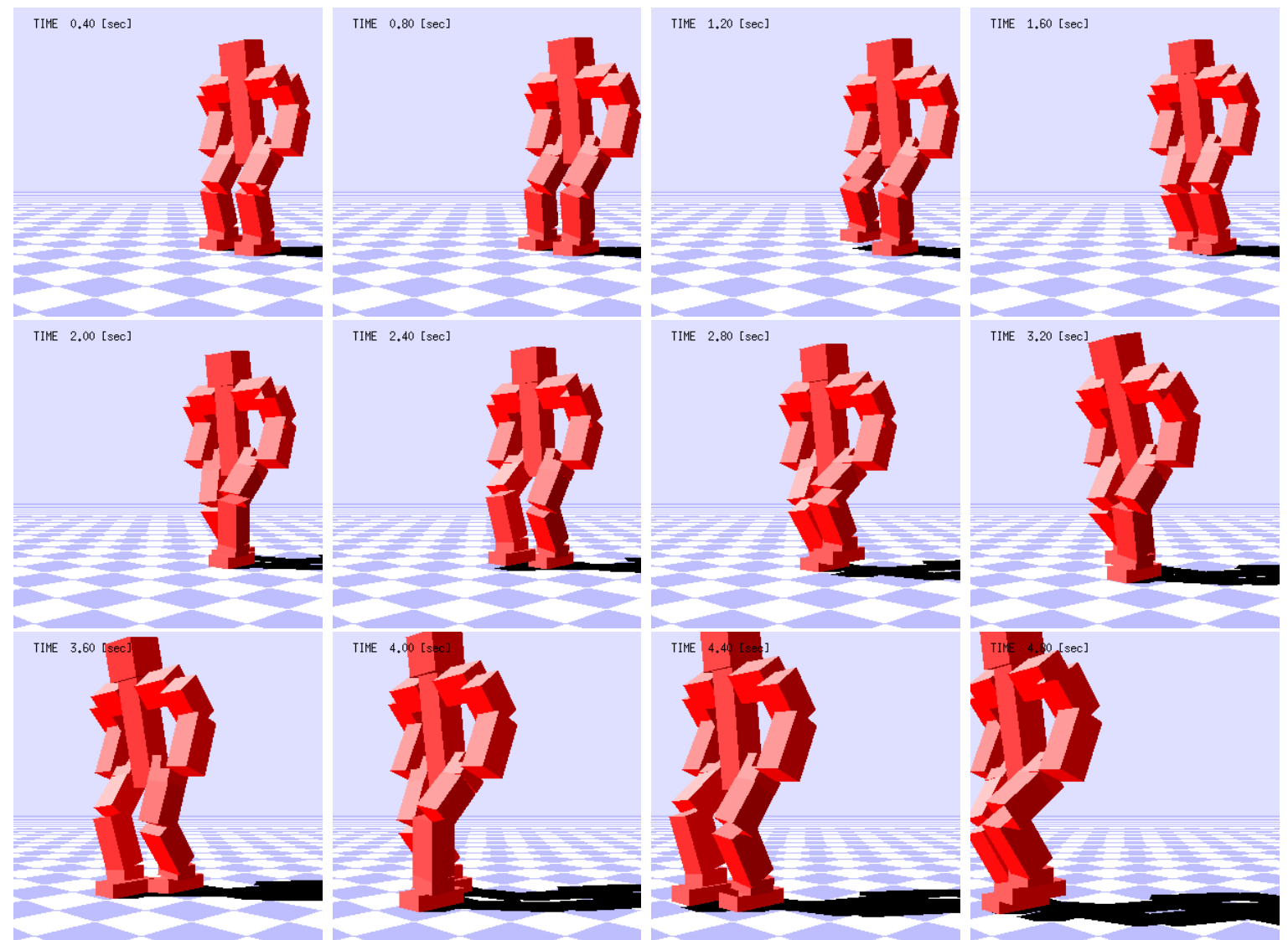

Fig. 7. Snapshots of biped walking simulation. (More details are found in the web, http://www.kawalab.dnj.ynu.ac.jp/)

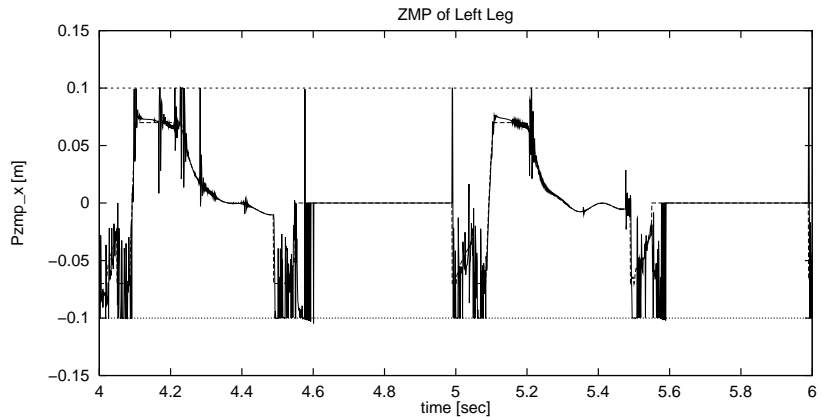

(a) ZMP of left foot in sagital plane.

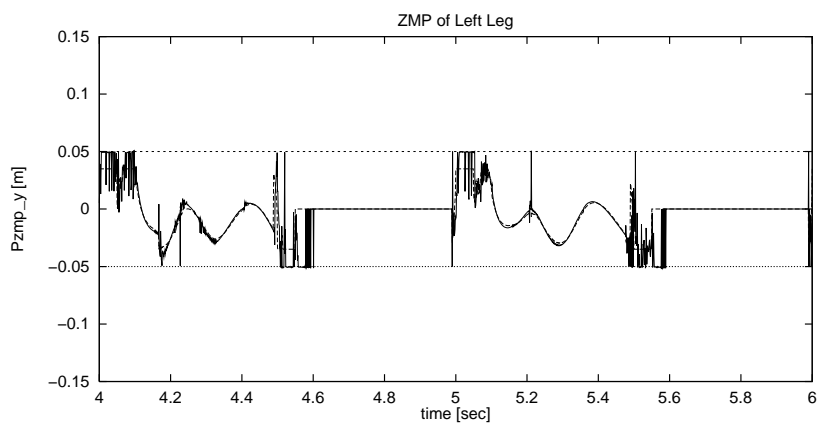

(c) ZMP of left foot in lateral plane.

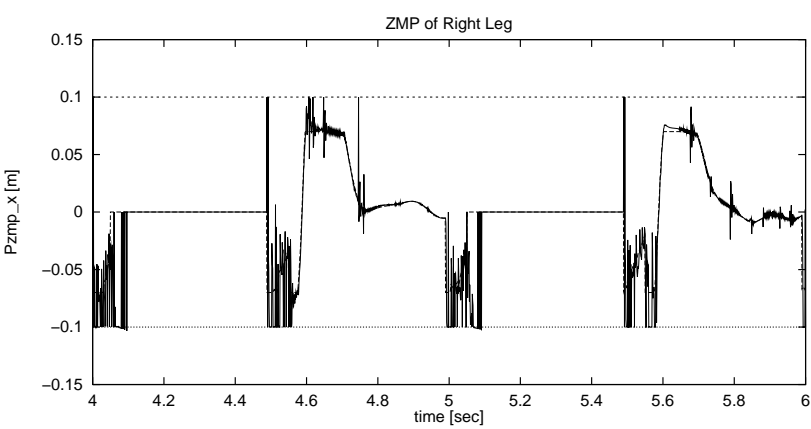

(b) ZMP of right foot in sagital plane.

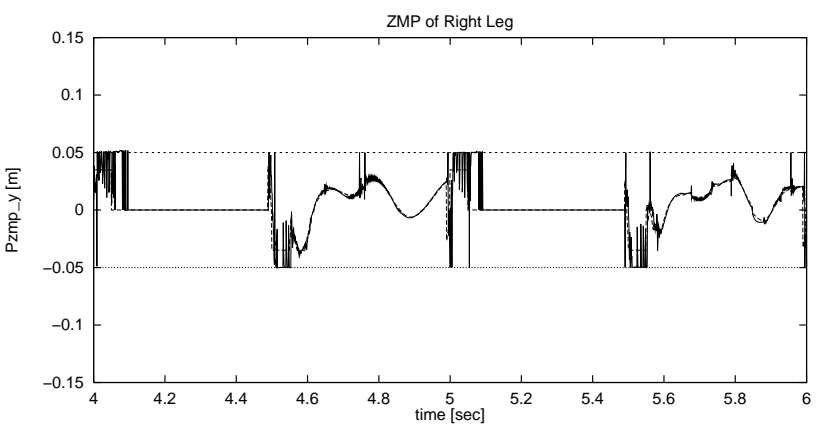

(d) ZMP of right foot in lateral plane.

Fig. 8. Trajectory of zero moment point. 


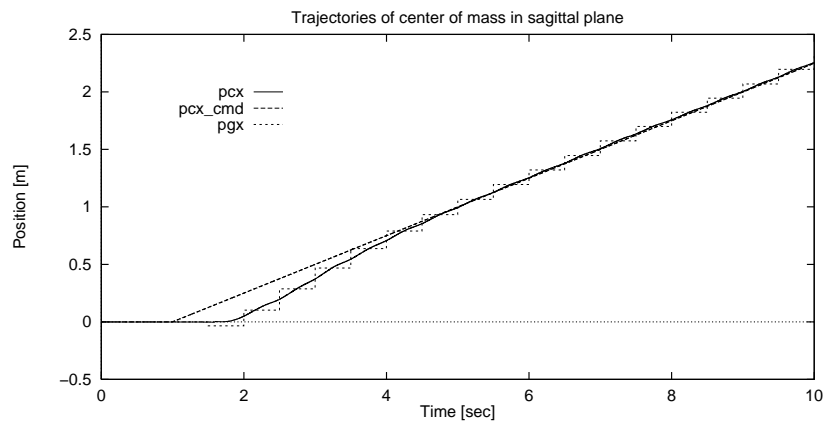

(a) In sagittal plane.

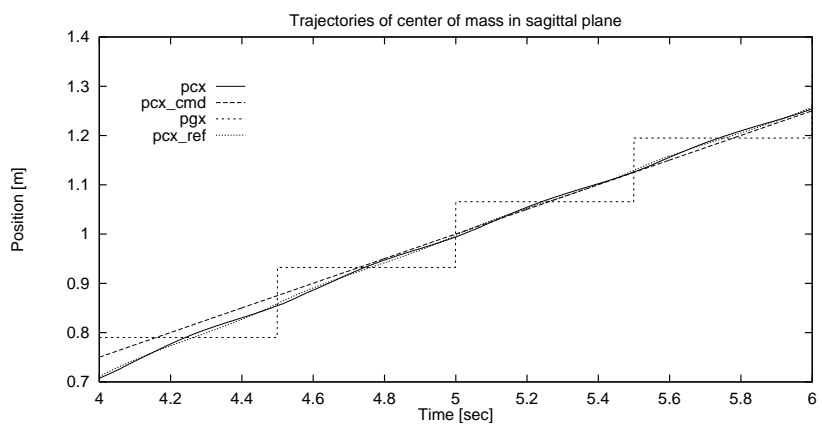

(c) In sagittal plane (magnified.)

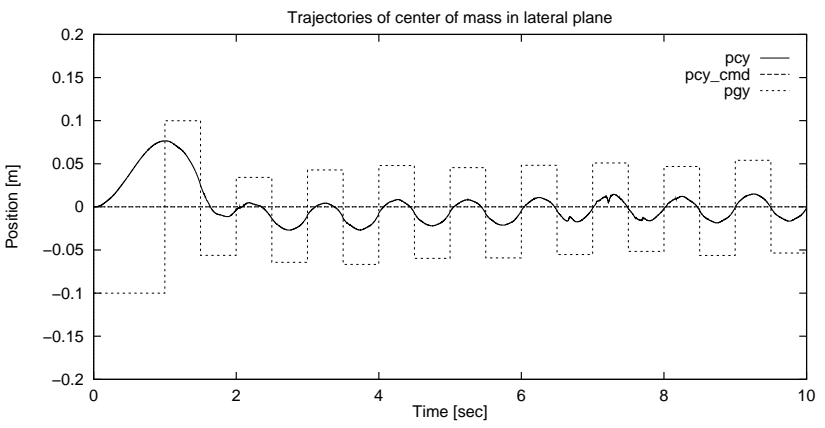

(b) In lateral plane.

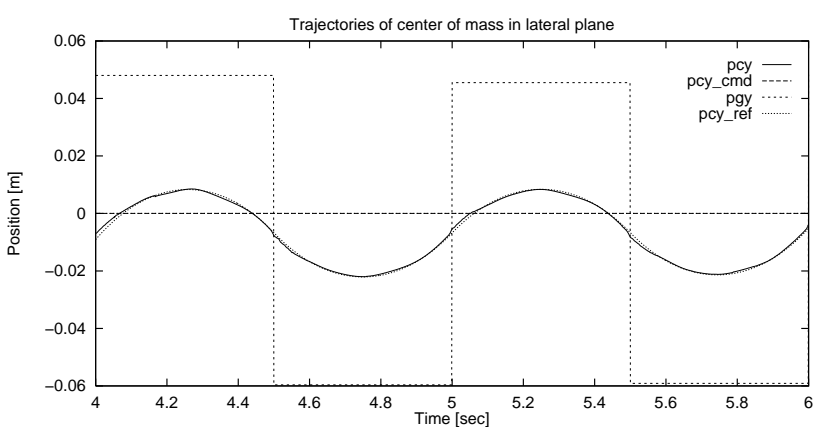

(d) In lateral plane (magnified.)

Fig. 9. Trajectory of center of mass of the robot

[14] Y. Fujimoto and A. Kawamura, "An Inertia Fluctuation Insensitive Robust Control of Robot Manipulators Based on a Combination of Inertia Torque Computation Filter and $H_{\infty}$ Control," Trans. of Institute of Electrical Engineers of Japan, vol. 117-D, no. 4, pp. 493-500, 1997. (in Japanese)

[15] Y. Fujimoto and A. Kawamura, "Proposal of Biped Walking Control Based on Robust Hybrid Position/Force Control," Proc. IEEE Int. Conf. on Robotics and Automation, pp. 2724-2730, 1996.

[16] Y. Fujimoto and A. Kawamura, "Biped Walking Control with Optimal Foot Force Distribution by Quadratic Programming," Proc. IEEE/ASME Int. Conf. on Advanced Intelligent Mechatronics, CD-ROM, 1997.

[17] Y. Fujimoto and A. Kawamura, "Robust Control of Biped Walking Robot with Yaw Moment Compensation by Arm Motion", Proc. Asian Control Conference, vol. 3, pp. 327-330, 1997.

[18] B. Nobel, Applied Linear Algebra, Prentice-Hall, Englewood Cliffs, N.J., 1969.

[19] J. Y. S. Luh, M. W. Walker, and R. P. C. Paul, "On-line Computational Scheme for Mechanical Manipulators," ASME J. Dynamic Systems, Measurement, Control, vol. 102, pp. 69-76, 1980 .

[20] M. W. Walker and D. E. Orin, "Efficient Dynamic Computer Simulation of Robotic Mechanisms," ASME J. Dynamic Systems, Measurement, Control, vol. 104, pp. 205-211, 1982.

[21] P. Lötstedt, "Numerical Simulation of Time-Dependent Contact and Friction Problems in Rigid Body Mechanics," SIAM J. Scientific and Statistical Computing, vol. 5, no. 2, pp. 370-393, 1984 .

[22] R. W. Cottle and G. B. Dantzig, "Complementary Pivot Theory of Mathematical Programming," Linear Algebra and Its Applications, vol. 1, pp. 103-125, 1968

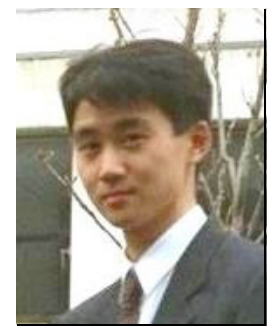

Yasutaka Fujimoto (S'93-M'98) was born in Kanagawa, Japan, in 1971. He received B.S., M.S., and Ph.D degrees in electrical engineering from Yokohama National University, Yokohama, Japan, in 1993, 1995, and 1998, respectively. He was a Research Fellow of the Japan Society for the Promotion of Science from 1995 to 1998.

In 1998 he joined the department of electrical engineering at Keio University, Yokohama, Japan as a research associate. His research interests are motion control and biped robots.

Dr. Fujimoto is a member of the IEE of Japan and the Robotics Society of Japan.

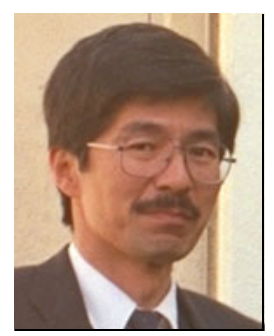

Atsuo Kawamura (S'77-M'81-SM'96) was born in Yamaguchi Prefecture, Japan, in 1953. He received the B.S., M.S., and Ph.D. degrees in electrical engineering from the University of Tokyo, Tokyo, Japan, in 1976, 1978, and 1981, respectively. In 1981 he joined the department of electrical and computer engineering at the University of Missouri-Columbia as a postdoctoral fellow, and was an assistant professor there from 1983 through 1986. In 1986 he joined the department of electrical and computer engineering at Yokohama National University, Yokohama, Japan as an associate professor, and became a professor in 1996. He is the department head from 1997 to 1998.

His interests are in power electronics, digital control, ultrasonic actuators, electric vehicles, robotics, and so on. He is a recipient of IEEE IAS Transactions Prize Paper Award in 1988, and IEE of Japan Transactions Prize Paper Award in 1996. He was the conference chairperson of IEEE/IAS and IEEJ/IAS joint Power Conversion Conference (PCC-Yokohama) in 1993. He is an associate editor of IEEE Power Electronics transactions. He will serve as the technical program chairman of Power Electronics Specialist Conference in 1998 (PESC'98).

Dr. Kawamura is a member of the IEE of Japan, IEEE, Robotics Society of Japan, the Institute of Electronics, Information and Communication Engineers, the Society of Instrument and Control Engineering. 